\title{
Susceptibility to Amoxicillin-Clavulanate-Induced Liver Injury Is Influenced by Multiple HLA Class I and II Alleles
}

\author{
M. ISABEL LUCENA, ${ }^{*}$ MARIAM MOLOKHIA, ${ }^{\ddagger}$ YUFENG SHEN, ${ }^{\S}$ THOMAS J. URBAN," GURUPRASAD P. AITHAL, ${ }^{\prime}$ \\ RAÚL J. ANDRADE, ${ }^{*}$ CHRISTOPHER P. DAY, " FRANCISCO RUIZ-CABELLO, ${ }^{* *, \neq \ddagger}$ PETER T. DONALDSON, \\ CAMILLA STEPHENS, ${ }^{*}$ MUNIR PIRMOHAMED, §§ MANUEL ROMERO-GOMEZ," II JOSE MARIA NAVARRO, "१ \\ ROBERT J. FONTANA, ${ }^{\# \#}$ MICHAEL MILLER, ${ }^{\star \star *}$ MAX GROOME, ${ }^{\star \star \star}$ EMMANUELLE BONDON-GUITTON, ${ }^{\neq \neq \neq}$

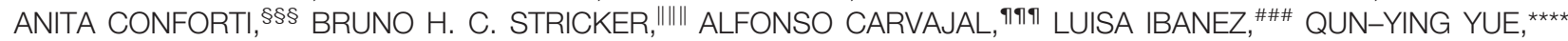

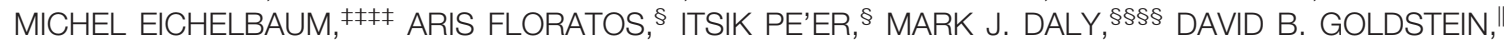

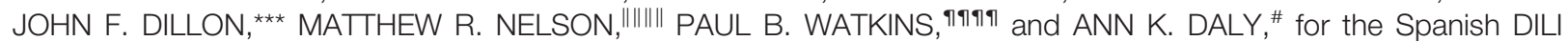 \\ Registry, EUDRAGENE, DILIN, DILIGEN, and International SAEC
}

\begin{abstract}
*Facultad de Medicina, Hospital Universitario Virgen de la Victoria, Campus Universitario s/n, Málaga and Centro de Investigación Biomédica en Red de Enfermedades Hepáticas y Digestivas (CIBERehd), Barcelona, Spain; ”Department of Primary Care \& Public Health Sciences, Kings College, London, United Kingdom; §Columbia University, New York, New York; "Duke University, Durham, North Carolina; "Nottingham Digestive Diseases Centre, NIHR Biomedical Research Unit, Nottingham, United Kingdom; *Institute of Cellular Medicine, Newcastle University, Newcastle upon Tyne, United Kingdom; **Hospital Universitario Virgen de las Nieves, Facultad de Medicina, Granada, Spain; ${ }^{\ddagger \ddagger}$ Red Genómica del Cáncer, Spain; §§ University of Liverpool, Liverpool, United Kingdom; IIIHospital Universitario de Valme, Sevilla, Spain; "19l Hospital Costa del Sol, Marbella, Málaga, Spain; \#\# University of Michigan Medical School, Ann Arbor, Michigan; ${ }^{* * * N i n e w e l l s ~ H o s p i t a l ~ a n d ~}$ Medical School, Dundee, United Kingdom; ${ }^{\ddagger \neq}$ Université de Toulouse, Toulouse, France; §§§University Hospital, Verona, Italy; IIIIIErasmus Medical Center, Rotterdam, The Netherlands; " $91 \%$ Universidad de Valladolid, Valladolid, Spain; "\#\#FICF (Catalan Institute of Pharmacology Foundation), Barcelona, Spain; ${ }^{* * * * M e d i c a l ~ P r o d u c t s ~}$ Agency, Uppsala, Sweden; ${ }^{\ddagger \neq \neq}$ Dr. Margarete Fischer-Bosch Institute for Clinical Pharmacology, Stuttgart, and University of Tuebingen, Germany; §§§ Harvard

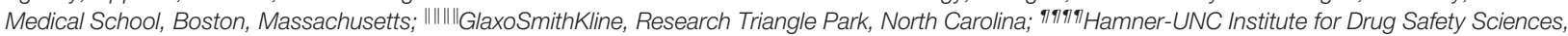
Research Triangle Park, North Carolina
\end{abstract}

BACKGROUND \& AIMS: Drug-induced liver injury (DILI), especially from antimicrobial agents, is an important cause of serious liver disease. Amoxicillin-clavulanate (AC) is a leading cause of idiosyncratic DILI, but little is understood about genetic susceptibility to this adverse reaction. METHODS: We performed a genome-wide association study using 822,927 single nucleotide polymorphism (SNP) markers from 201 White European and US cases of DILI following AC administration (AC-DILI) and 532 population controls, matched for genetic background. RESULTS: AC-DILI was associated with many loci in the major histocompatibility complex. The strongest effect was with an HLA class II SNP (rs9274407, $P=4.8 \times$ $\left.10^{-14}\right)$, which correlated with rs3135388, a tag SNP of HLA$D R B 1 * 1501-D Q B 1 * 0602$ that was previously associated with AC-DILI. Conditioned on rs3135388, rs9274407 is still significant $\left(P=1.1 \times 10^{-4}\right)$. An independent association was observed in the class I region ( $\left.\mathrm{rs} 2523822, P=1.8 \times 10^{-10}\right)$, related to $H L A-A * 0201$. The most significant class I and II SNPs showed statistical interaction $(P=.0015)$. High-resolution HLA genotyping (177 cases and 219 controls) confirmed associations of $H L A-A^{*} 0201\left(P=2 \times 10^{-6}\right)$ and HLA-DQB1*0602 $\left(P=5 \times 10^{-10}\right)$ and their interaction $(P=$ .005). Additional, population-dependent effects were observed in HLA alleles with nominal significance. In an analysis of autoimmune-related genes, rs2476601 in the gene PTPN22 was associated $\left(P=1.3 \times 10^{-4}\right)$. CONCLUSIONS: Class I and II HLA genotypes affect susceptibility to AC-DILI, indicating the importance of the adaptive immune response in pathogenesis. The HLA genotypes identified will be useful in studies of the pathogenesis of AC-DILI but have limited utility as predictive or diagnostic biomarkers because of the low positive predictive values.
Keywords: Hepatotoxicity; Genome-Wide Association Study; GWAS; Pharmacogenomics.

Tdiosyncratic liver toxicity because of a prescribed drug is usually referred to as drug-induced liver injury (DILI). Most DILI involves reactions that appear unrelated to drug dose or concentration. Although comparatively rare, DILI is a serious clinical problem with up to $10 \%$ of cases with simultaneous severe elevations in alanine transaminase (ALT) and bilirubin developing liver failure. ${ }^{1}$ A prospective study estimated the standardized incidence rate of symptomatic hepatic adverse drug reactions at 8.1 per 100,000 people in France. ${ }^{2}$ In the United States, $13 \%$ of acute liver failure cases are because of idiosyncratic hepatotoxicity with $75 \%$ of those dying or requiring emergency liver transplantation. ${ }^{3}$

Amoxicillin-clavulanate $(\mathrm{AC})$ is among the most commonly prescribed antimicrobials worldwide. This drug is generally well tolerated, and, whereas liver injury can occur rarely, the overall risk benefit is favorable. DILI following AC administration (AC-DILI), which appears to be

Abbreviations used in this paper: AC, amoxicillin-clavulanate; AC-DILI, DILI following AC administration; ADME, drug absorption, distribution, metabolism, and excretion; ALP, alkaline phosphatase; ALT, alanine transaminase; AST, aspartate aminotransferase; $\mathrm{Cl}$, confidence interval; CIOMS, Council for International Organizations of Medical Science; DILI, drug-induced liver injury; DILIN, drug-induced liver injury network; LD, linkage disequilibrium; MHC, major histocompatibility complex; NPV, negative predictive value; OR, odds ratio; PBC, primary biliary cirrhosis; POPRES, Population Reference Sample; Q-Q, quantile-quantile; SNP, single nucleotide polymorphism; ULN, upper limit of normal. (C) 2011 by the AGA Institute 0016-5085/ \$36.00 doi:10.1053/j.gastro.2011.04.001 
primarily due to the clavulanate component, ${ }^{4}$ is an important cause of idiosyncratic DILI in the United States and Europe, ${ }^{5-7}$ and represents $17 \%$ of all DILI-related hospitalizations. ${ }^{6,8}$ Whereas most patients with AC-DILI make a full recovery, ${ }^{5,9}$ cases of acute liver failure leading to death or liver transplantation have been reported. ${ }^{10}$ The mechanism of AC-DILI is unknown. Three previous studies from Northwestern Europe ${ }^{11-13}$ reported an association between AC-DILI and the HLA class II allele $D R B 1 * 1501$, with odds ratios (OR) ranging between 2.6 and 10. A further study of 27 Spanish cases did not observe a significant association with $D R B 1 * 15$ but did report a significantly higher frequency of $D Q B 1 * 06 .{ }^{14}$ The differences in findings between the Spanish and Northwestern European studies may be due to use of lowresolution genotyping, population-specific linkage-disequilibrium patterns, population stratification, or to a larger proportion of hepatocellular cases in the Spanish study. ${ }^{15}$

We conducted a genome-wide association (GWA) study to investigate whether additional common genetic variants affect susceptibility to AC-DILI. A group of wellphenotyped cases $(\mathrm{n}=201)$ with high causality scores were assembled from several multicenter collections together with 532 genetically matched population controls. Our study confirms the DRB $1 * 1501$ association and identifies additional HLA class I and II associations.

\section{Materials and Methods}

\section{Case Recruitment}

Cases $(\mathrm{n}=211)$ were recruited in 4 separate studies (DILIGEN, ${ }^{16}$ Spanish DILI Registry, ${ }^{6}$ Drug-induced liver injury network [DILIN], ${ }^{7}$ and EUDRAGENE ${ }^{17}$ ) that used similar inclusion criteria. All participants provided written informed consent, and each study had been approved by the appropriate ethical review boards.

DILIGEN. Between October 2004 and April 2009, 78 cases of AC-DILI of European origin from centers throughout the United Kingdom were collected. Inclusion criteria were suspected liver injury because of AC with either (1) clinically apparent jaundice or bilirubin $>2.4$ $\mathrm{mg} / \mathrm{dL}$ (after exclusion of cases because of hemolysis), (2) ALT $>5 \mathrm{X}$ upper limit of normal (ULN), or (3) alkaline phosphatase (ALP) $>2 \mathrm{X}$ ULN plus bilirubin above ULN. Cases were identified by searching histologic databases and discharge records at UK regional liver units for cases of DILI or cholestasis/hepatitis of unknown etiology. Direct contact with gastroenterologists was also made by advertising the study through professional societies.

EUDRAGENE. Adult ( $\geq 18$ years of age) AC-DILI cases identified retrospectively and prospectively from European pharmacovigilance center adverse drug reaction reports were collected from November 2006 to August 2009. Of the 25 cases included in this study, 15 were from Spain, 7 from France, and 3 from Italy.

Case definitions included (1) acute liver injury defined as presenting symptoms suggestive of liver disorder (nau- sea, vomiting, abdominal pain, and/or jaundice), referred to a specialist, or admitted to hospital; (2) ALT $>2 \mathrm{X}$ ULN; or (3) aspartate aminotransferase (AST), ALP, and total bilirubin $>2 \mathrm{X}$ ULN.

Spanish DILI registry. Fifty-two cases of AC-DILI patients $\geq 18$ years of age were selected from those submitted to the Spanish DILI Registry, a collaborative network set up in 1994 to prospectively identify cases of DILI in a standardized manner. Inclusion laboratory criteria for AC-DILI cases in this study were (1) clinically apparent jaundice or bilirubin $>2.4 \mathrm{mg} / \mathrm{dL}$ (after exclusion of cases because of hemolysis), (2) ALT $\geq 5 \mathrm{X}$ ULN, or (3) ALP $\geq 2 \mathrm{X}$ ULN. Detailed description of the operational structure of the registry, data recording, and case ascertainment has been reported elsewhere. ${ }^{6}$

DILIN. Details of the DILIN prospective study have been described previously. ${ }^{18} \mathrm{~A}$ total of 65 eligible AC-DILI cases was recruited between August 2004 and April 2009 from 5 DILIN clinical sites in the United States. Of these, 56 cases of European ancestry and $\geq 18$ years of age were included in the current study. Inclusion laboratory criteria were (1) serum AST or ALT $>5 \mathrm{X}$ ULN on 2 separate occasions, (2) serum ALP $>2 \mathrm{X}$ ULN on 2 consecutive occasions, or (3) serum total bilirubin $>2.4$ $\mathrm{mg} / \mathrm{dL}$ in the absence of a competing cause of hyperbilirubinemia. Patients were excluded if there was known or suspected acetaminophen overdose, if there was a history of bone marrow or liver transplant prior to DILI onset, or if there was a prior history of immune-related liver disease such as autoimmune hepatitis.

\section{Causality Assessment}

Diagnosis of DILI was done by expert hepatologists at each of the collaborating centers. In addition, the cases were evaluated by application of the Council for International Organizations of Medical Science (CIOMS) scale, also called the Roussel Uclaf Causality Assessment Method (RUCAM). The pattern of liver injury was classified according to the International Consensus Meeting Criteria. ${ }^{19}$ Only cases having at least possible causality (score $\geq 3$ ) were included in the study.

\section{DNA Preparation From Cases}

For DILIGEN and Spanish DILI Registry cases, DNA was prepared as described previously. ${ }^{14,16}$ EUDRAGENE DNA was extracted at Erasmus Medical Centre genotyping laboratory, The Netherlands, using standard procedures. DILIN DNA was extracted from lymphocytes and stored at the National Institute of Diabetes and Digestive and Kidney Diseases biosample repository at Rutgers University, Piscataway, NJ.

\section{Controls}

Genotyped controls $(\mathrm{n}=532)$ from the Population Reference Sample (POPRES) ${ }^{20}$ were matched to the cases using principal component analysis. Based on the first 2 principal components that capture much of the genetic substructure among Europeans, 306 controls were

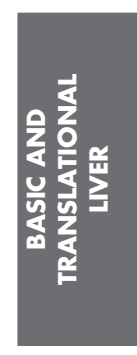


Table 1. Clinical Characteristics of AC-DILI Cases

\begin{tabular}{|c|c|c|c|c|c|}
\hline Cohorts & DILIGEN & EUDRAGENE & Spanish DILI Registry & DILIN & All \\
\hline No. & 77 & 19 & 49 & 56 & 201 \\
\hline \multicolumn{6}{|l|}{ Sex, n (\%) } \\
\hline $\mathrm{M}$ & 39 (51) & $11(58)$ & $27(55)$ & $36(64)$ & $113(56)$ \\
\hline $\mathrm{F}$ & 38 (49) & $8(42)$ & $22(45)$ & $20(36)$ & $88(44)$ \\
\hline \multicolumn{6}{|l|}{ Age, $y$} \\
\hline Mean $( \pm S D)$ & $61( \pm 14)$ & $68( \pm 14)$ & $60( \pm 18)$ & $59( \pm 14)$ & $61( \pm 14)$ \\
\hline \multicolumn{6}{|l|}{ Total days on drug } \\
\hline Mean $( \pm$ SD) & $8( \pm 6)$ & $7( \pm 5)$ & $11( \pm 9)$ & $12( \pm 8)$ & $10( \pm 7)$ \\
\hline \multicolumn{6}{|l|}{ Days to DILI onset } \\
\hline Mean ( \pm SD) & $15( \pm 14)$ & $11( \pm 9)$ & $16( \pm 12)$ & $32( \pm 19)$ & $20( \pm 17)$ \\
\hline \multicolumn{6}{|c|}{ Pattern of liver injury, n (\%) } \\
\hline Cholestatic & $36(47)$ & $6(32)$ & $17(35)$ & $20(36)$ & 79 (39) \\
\hline Mixed & $18(23)$ & $5(26)$ & $17(35)$ & $23(41)$ & $63(31)$ \\
\hline Hepatocellular & $19(25)$ & 0 & $15(31)$ & $10(18)$ & $44(22)$ \\
\hline Unknown & $4(5)$ & $8(42)$ & 0 & $3(5)$ & $15(7)$ \\
\hline \multicolumn{6}{|c|}{ Causality (CIOMS), n (\%) } \\
\hline Unlikely & 0 & 0 & 0 & 0 & 0 \\
\hline Possible & $7(9)$ & $1(5)$ & 0 & $6(11)$ & $14(7)$ \\
\hline Probable & $25(32)$ & $12(63)$ & $23(47)$ & $37(66)$ & $97(48)$ \\
\hline Highly probable & $45(58)$ & $6(32)$ & $26(53)$ & $13(23)$ & $90(45)$ \\
\hline \multicolumn{6}{|c|}{ Peak Bilirubin, $m g / d L$} \\
\hline Mean $( \pm \mathrm{SD})$ & $12.5( \pm 9.8)$ & $10.8( \pm 12.2)$ & $12.3( \pm 9.5)$ & $16.5( \pm 11.8)$ & $13.5( \pm 10.6)$ \\
\hline \multicolumn{6}{|l|}{ Peak ALT, $U / L$} \\
\hline Mean $( \pm$ SD) & $455( \pm 465)$ & $342( \pm 268)$ & $548( \pm 668)$ & $575( \pm 713)$ & $500( \pm 584)$ \\
\hline \multicolumn{6}{|l|}{ Peak ALP, U/L } \\
\hline Mean ( \pm SD) & $453( \pm 253)$ & $438( \pm 171)$ & $417( \pm 286)$ & $456( \pm 293)$ & $444( \pm 268)$ \\
\hline
\end{tabular}

F, female; M, male; SD, standard deviation.

selected as Northwestern Europeans (predominantly POPRES UK), and 160 controls were selected as Spanish (predominantly POPRES Spanish and Portuguese).

\section{Genotyping}

Genome-wide analysis. Genome-wide genotyping of the European DILI cases and POPRES controls was performed by Expression Analysis, Inc (Durham, NC) and of the US cases by the Center for Human Genome Variation, Duke University. All subjects were genotyped using the Human1M-Duo BeadChip (Illumina, San Diego, CA), containing 1,072,820 markers. A total of 822,927 markers, 201 cases (77 DILIGEN, 19 EUDRAGENE, 49 Spanish DILI Registry, and 56 DILIN) and 532 controls passed quality control (see Supplementary Materials and Methods).

HLA genotyping. High-resolution genotyping of $H L A-A,-B,-D R B 1,-D Q A 1$, and $-D Q B 1$ was performed on a subset of genotyped subjects, including 182 cases (74 DILIGEN, 49 Spanish DILI Registry, and 59 DILIN) and 219 POPRES controls (genetically matched to cases as described above) by Histogenetics (Ossining, New York) as described in the Supplementary Materials and Methods.

\section{Statistical Analysis}

The statistical association of each marker in the GWA study was determined by logistic regression with gender and first 2 principal components as covariates under an additive model and Fisher exact allelic test within subpopulation analyses. GWA analyses were carried out with PLINK. $^{21}$ We inferred the genomic inflation factor by con- verting $P$ values from regression test to $\chi^{2}$ values then taking the median (0.4687) divided by the expected median value (0.456) of $\chi^{2}$ distribution with 1 degree of freedom.

HLA haplotypes were inferred using Beagle version 2.1.22 Associations of HLA alleles were tested using logistic regression, including the first 2 principal component scores as covariates to control for population stratification. To test for independent effects within regions having multiple associated variants, we included 1 or more variants as a covariate and tested the significance of other variants by adding them stepwise. All detailed analyses were performed with R. ${ }^{23} \mathrm{Ad}-$ ditional statistical analyses of HLA alleles were conducted by logistic regression using glm function (generalized linear model) and heterogeneity testing with meta.MH function (Mantel-Haenszel meta-analysis) from the rmeta package (http://cran.r-project.org/web/packages/rmeta/index.html) adjusting for gender and principal components when feasible.

\section{Results}

\section{Clinical Characteristics of the Cases}

Clinical details of the 201 cases included in this study are summarized in Table 1. As in previous studies of AC-DILI, there were more male than female cases. The average age at onset was $61 \pm 14$ years. Four cases underwent liver transplantation. Most cases (70\%) were characterized as cholestatic or mixed at presentation, and $88 \%$ had bilirubin levels $>2.4 \mathrm{mg} / \mathrm{dL}$. Most causality scores (93\%) suggested that DILI was either probably or highly likely because of AC. There were some significant differences in the pattern of disease and other phenotypic 
characteristics among the 4 collections (Supplementary Table 1).

\section{Genome-Wide Analysis}

GWA analysis was carried out on 201 DILI cases and 532 controls for 822,927 markers that passed quality control. Principal component analysis showed that all cases clustered within Northwestern, Western, and Southern Europe (Supplementary Figure 1), consistent with previous studies on Europeans. ${ }^{24}$ Based on these genetic patterns, the US-derived DILIN samples were predominantly of Northwestern European origin with genotype patterns correlating well with both UK controls and cases. The genomic inflation factor $(\lambda)$ of our study was 1.03 , which indicates no problems with population stratification.

Case-control association analysis revealed 1 marked peak of association with several dozen genome-wide significant SNPs within the major histocompatibility complex (MHC) on chromosome 6p21.3 (Figure 1A). Quantile-quantile (Q-Q) plots (Supplementary Figure 2) suggested that no SNPs outside the MHC region showed a genome-wide significant association with DILI. The most significant associations localized within the class II and class I regions (Figure $1 B$ ). The top SNP associated with AC-DILI was rs9274407 $\left(P=4.8 \times 10^{-14}\right.$ with an estimated additive OR of 3.1 (95\% confidence interval [CI]: 2.3-4.2); Table 2). This SNP is located within the HLA-DQB1 gene and had relatively high linkage disequilibrium (LD) $\left(r^{2}=0.76\right)$ with rs3135388, one of the other top associated SNPs that is strongly correlated with (ie, a tag for) $D R B 1 * 1501$ and the haplotype DRB1*1501$D Q B 1 * 0602 .{ }^{25}$ The top SNP (rs9274407) was significantly associated conditioned on the tag SNP (rs3135388) $(P=$ $.00011)$, whereas rs3135388 was not significantly conditioned on rs9274407 $(P=.93)$.

Several SNPs from the HLA class I region were also genome-wide significant, the most significant being rs2523822 $\left(P=1.8 \times 10^{-10}\right.$, OR, 2.3 ; 95\% CI: $\left.1.8-2.9\right)$, previously reported as a tag for $A^{*} 0201 .^{25}$ Conditioning on rs9274407, the association peak within class I remained genome-wide significant, whereas none of the other class II region SNPs remained significantly associated (Figure 1C, Supplementary Figure 2, Table 2). We found a statistically significant interaction $(P=.0015$, OR, 2.3; 95\% CI: 1.4-3.8) between rs9274407 and rs2523822, indicating that the increased risk when both minor alleles were present was larger than expected based on their individual effects. Conditioned on both rs9274407 and rs2523822, no additional remarkable associations within the MHC were observed (Figure $2 D$ ).

A total of 14 of the 201 cases in the study had shown "possible" rather than "probable" or "highly probable" causality when scored by the CIOMS system. ${ }^{19}$ To assess whether exclusion of these cases from the analysis would affect the overall findings, we recalculated $P$ values for the 2 SNPs with the lowest $P$ values (Table 2). No significant alteration in these $P$ values was seen (data not shown).
To consider the potential for country-specific effects, genome-wide analyses were carried out on 3 separate subgroups within the original 201 cases, namely 74 UK, 51 US cases with Northwestern European genetic backgrounds, and 46 Spanish DILI Registry cases with Spanish genetic background (Supplementary Figure 2). Appropriately matched POPRES controls (306 Northwestern European for the United Kingdom and United States and 160 Spanish and Portuguese) were used. As with the combined samples, no SNPs outside the MHC were genome-wide significant, and most significant SNPs in the combined sample showed similar estimates of effects within each of the 3 groups (Table 3). Conditioning analysis on top SNPs, similar to that performed for the entire group shown in Table 2, was also performed on the different groups (Table 3). The association of rs9274407 conditioned on rs 3135388 was observed only in the UK group with statistical significance $\left(P=7.3 \times 10^{-5}\right)$. Rs3135388 was not significantly associated conditioned on rs9274407 consistently in all 3 groups, but rs 2523822 was significantly associated conditioned on either rs9274407 or rs3135388 in all 3 groups. The interaction between the class I rs252382 and class II rs9274407, which was significant for the entire group, was also significant in the UK cases alone $(P=.013)$ but not in the US and Spanish cases $(P=.14$ and $P=.31$, respectively).

Although the Q-Q plots (Supplementary Figure 3) suggested that no SNPs outside the MHC region were significantly associated with AC-DILI genome wide, we also assessed whether there was any indication of a contribution from either genes concerned with drug absorption, distribution, metabolism and excretion $(\mathrm{ADME})^{26}$ or, in view of the strong HLA associations, non-HLA genes known to be involved in autoimmune diseases generally. ${ }^{27}$ We extracted all SNPs within 10 kilobase for a list of 130 ADME genes. ${ }^{26}$ A total of 4961 such SNPs were included in our analysis. Figure $2 A$ shows the Q-Q plot of $P$ values for these SNPs and indicates that no significant associations occur. For the autoimmune disease genes, we extracted SNPs associated with these diseases with reported $P$ value smaller than $5 \times 10^{-8}$ (see Figure $2 B$ ). ${ }^{27}$ Among these SNPs (213 in total), 158 had been genotyped and analyzed in our study. Figure $2 B$ shows the Q-Q-plot of $P$ values of these SNPs, excluding SNPs from the MHC region. We found 2 SNPs, rs2476601 and rs6679677, in strong LD with each other, to be associated with AC-DILI (for rs2476601, $P=1.3 \times 10^{-4}$, OR, 2.1; 95\% CI: 1.5-3.2) (Supplementary Table 2). Rs2476601 is a nonsynonymous SNP in PTPN22, the gene encoding the lymphoid-specific protein tyrosine phosphatase, nonreceptor type 22 involved in T cell-receptor signaling, and has been reported to be associated with multiple autoimmune diseases. ${ }^{28}$ Although the association was not genome-wide significant, it had a $P$ value of .023 after Bonferroni correction for all the published GWA associations with autoimmune diseases that were genotyped in our study. ${ }^{27}$ The association of the PTPN22 SNPs with DILI appeared stronger in 

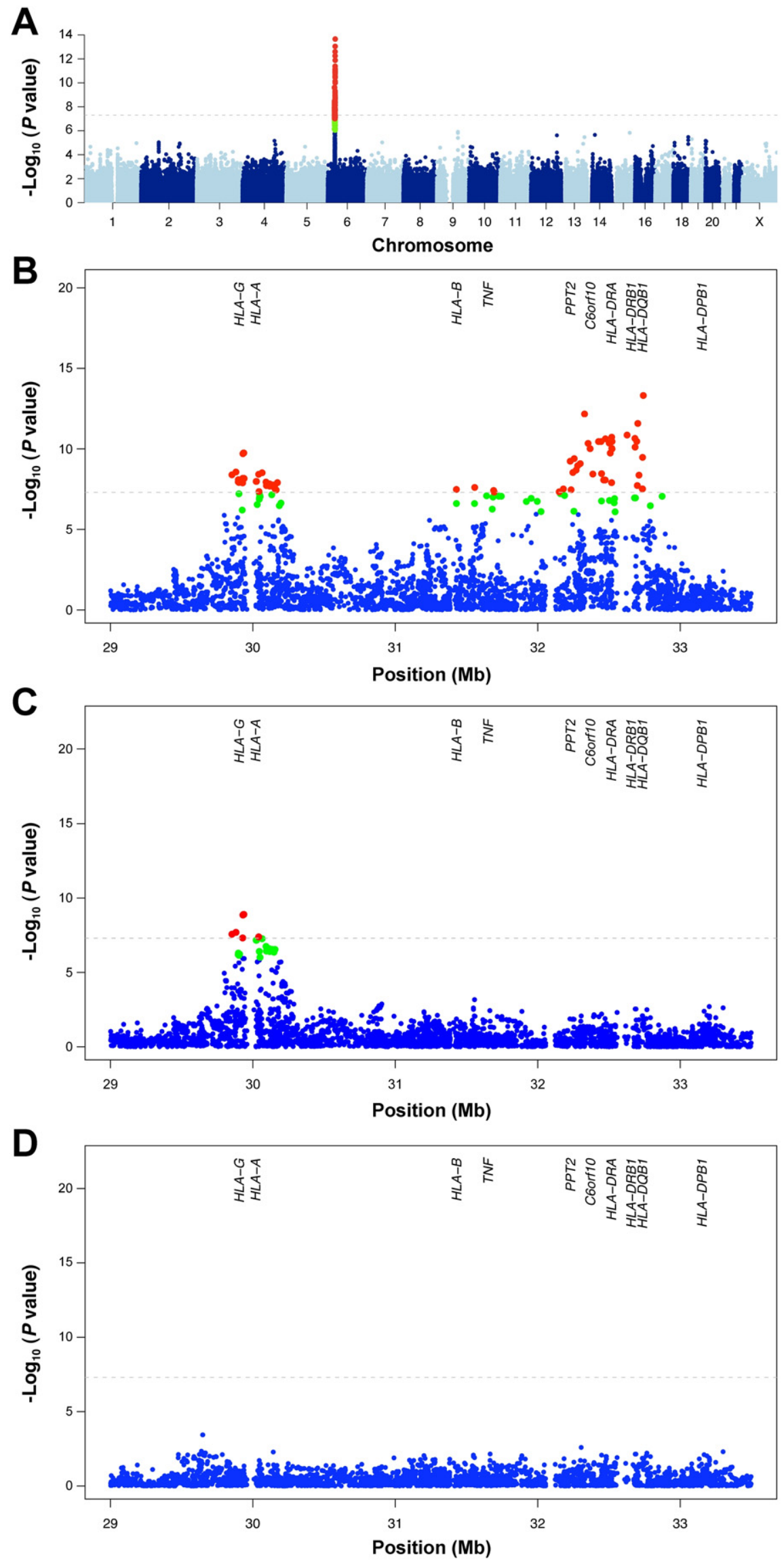

Figure 1. AC-DILI genome-wide association results. Each point represents association analysis results for a single SNP with chromosome position on the $x$-axis and $-\log _{10} P$ value on the $y$-axis. All 201 DILI cases and 532 population controls were included in the analysis. SNPs with $P$ values smaller than $10^{-6}$ and $10^{-7}$ are highlighted in green and red, respectively. Panel $A$ shows the results for the entire genome. Panels $B-D$ show enlarged sections of the $\mathrm{MHC}$ region. Panel $B$ is an enlargement of the results presented in $A$, panel $C$ shows the analysis of each SNP in this chromosome region conditioned on the top class II SNP (rs9274407), and panel $D$ shows analysis of each SNP conditioned on the top class I and II SNPs (rs9274407 and rs2523822). Positions of a range of $\mathrm{MHC}$ genes are shown in $B-D$. 
Table 2. Summary of Top Associated SNPs From the Genome-Wide Analysis Together With Conditioning Analysis

\begin{tabular}{|c|c|c|c|c|c|c|c|c|}
\hline SNPS & HLA allele tagged & $\begin{array}{l}\text { HLA } \\
\text { class }\end{array}$ & Chr & $\begin{array}{l}\text { Position } \\
\text { (build 36) }\end{array}$ & $P$ value & OR $(95 \% \mathrm{Cl})$ & $\begin{array}{l}P \text { value conditioned } \\
\text { on rs } 9274407\end{array}$ & $\begin{array}{c}P \text { value conditioned } \\
\text { on rs3135388 }\end{array}$ \\
\hline rs9274407 & & II & 6 & 32740809 & $4.8 \times 10^{-14}$ & $3.1(2.3-4.2)$ & N/A & .00011 \\
\hline rs9267992 & & II & 6 & 32328375 & $6.8 \times 10^{-13}$ & $3.1(2.3-4.2)$ & .07 & .0034 \\
\hline rs3135388 & $D R B 1 * 1501-D Q B 1 * 0602$ & II & 6 & 32521029 & $3.5 \times 10^{-11}$ & $2.8(2.1-3.8)$ & .93 & N/A \\
\hline rs2523822 & $H L A-A * 0201$ & 1 & 6 & 29936639 & $1.8 \times 10^{-10}$ & $2.3(1.8-2.9)$ & $1.2 \times 10^{-9}$ & $2.1 \times 10^{-10}$ \\
\hline
\end{tabular}

NOTE. The data shown are for all 201 drug-induced liver injury cases and 532 controls.

Chr, chromosome; N/A, not applicable.

the UK and US cases than in those from Spain (Supplementary Table 2).

\section{HLA Analysis}

To investigate further the observed associations within the MHC, high-resolution genotyping of HLA-A, $-B,-D R B 1,-D Q A 1$, and $-D Q B 1$ was performed on a subset of 177 cases and 219 genetically matched controls. The relationship between HLA genotypes and the top associated SNPs within Northwestern European and Spanish cases and controls was investigated (Supplementary Table 3). There were substantial differences in LD between Northwestern Europeans and Spanish for rs9274407 and $D Q B 1 * 0602$ and for $\operatorname{rs} 2523822$ and $A^{*} 0201$ that were reflected in subsequent associations. In particular, rs $2523822 / C$ showed poor correlation with $A^{*} 0201$ in Spanish, especially among cases $\left(r^{2}=0.64\right)$ compared with Northwestern Europeans ( $r^{2}=0.96$ for cases) (Supplementary Table 3). DQB1*0602 was the most significantly associated HLA allele with DILI overall $(P=1.4 \times$ $10^{-6}$, OR, 3.3; 95\% CI: $\left.2.0-5.7\right)$ and within each group. $D R B 1 * 1501$ was in near-perfect LD with $D Q B 1 * 0602$ in both Northwestern European and Spanish cases and controls, and the association of either allele with AC-DILI was statistically similar. A summary of key findings conditioned on $D Q B 1^{*} 0602$ is provided in Table 4. $D Q A 1 * 0102$, an allele included in but not exclusive to the $D R B 1 * 1501$ $D Q B 1 * 0602$ haplotype, was slightly more significant than $D Q B 1 * 0602$ in Northwestern Europeans but not in Spanish. Conditioned on $D Q B 1{ }^{*} 0602, D Q B 1{ }^{*} 0402$ was associated in Northwestern Europeans with nominal significance, but not in Spanish. This is consistent with the SNP results, where rs9274407 conditioned on rs3135388 was an indicator of a $D Q B 1 * 0402$ association in Northwestern Europeans (Supplementary Table 3). Unlike its tag SNP (rs3135388), DQB1*0602 was associated in the Spanish group conditioned on rs9274407 with nominal significance $(P=.01)$.

The class I allele $A^{*} 0201$ was associated $\left(P=2 \times 10^{-6}\right.$, OR, 2.2; 95\% CI: 1.6-3.2) with DILI in the combined cases. Consistent with the SNP results, there was a significant statistical interaction between $A^{*} 0201$ and $D Q B 1 * 0602$ in the combined cases $(P=.0048$, OR, 3.3; 95\% CI: $1.4-7.7)$ together with the UK and US cases individually $(P=.012$, OR, 3.9; 95\% CI $1.4-11$ and $P=.03$, OR, 5.4; 95\% CI: $1.2-25)$. However, $A^{*} 0201$ was not as strongly associated in Spanish as in Northwestern Europeans. A test of het-
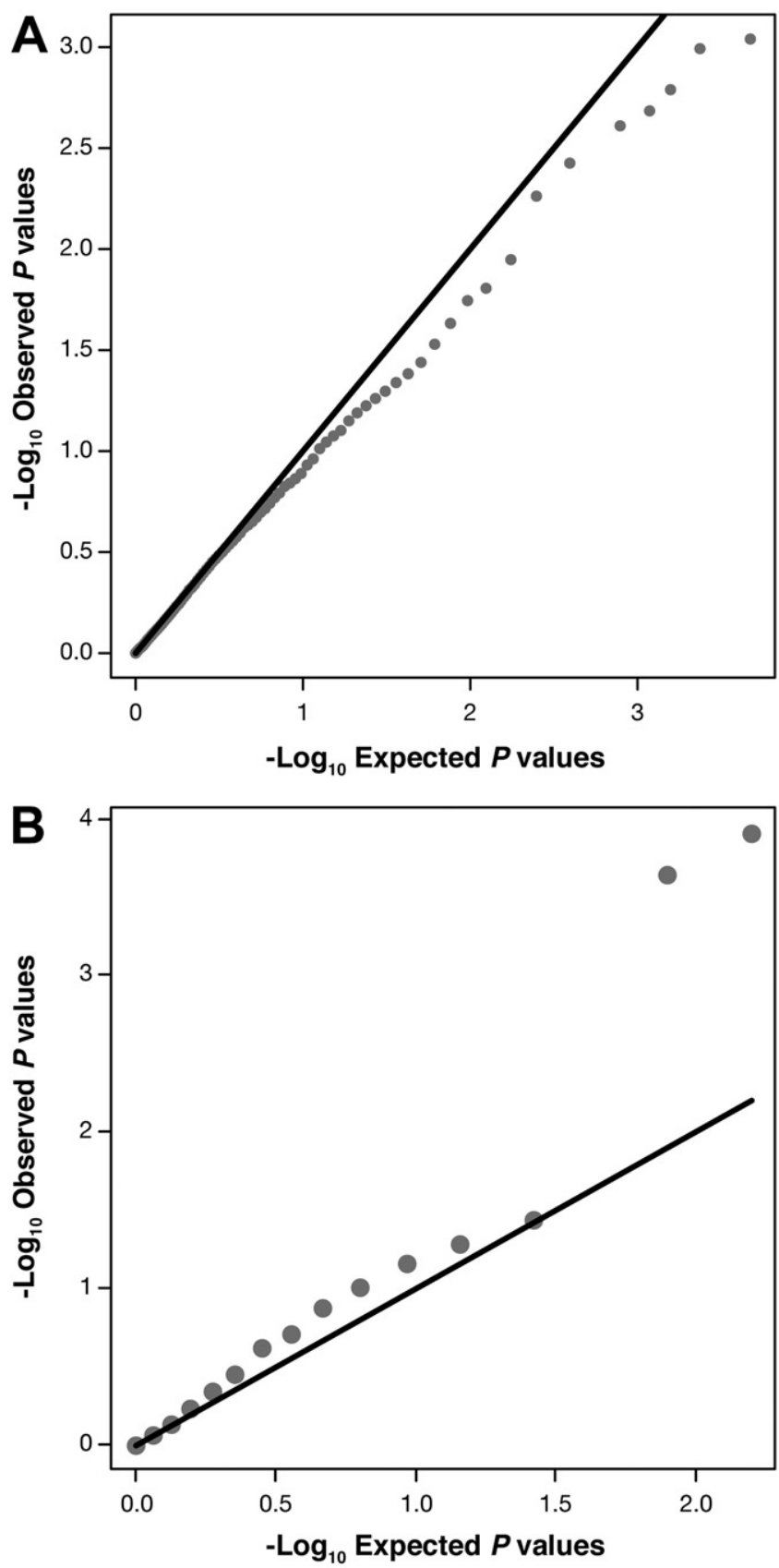

Figure 2. Additional $Q-Q$ plots for $A D M E$ and autoimmune genes. Panel A shows a Q-Q plot for ADME genes and panel B a Q-Q plot for GWAS hits for autoimmune disease genes. The autoimmune disease SNPs studied related to type 1 diabetes, Crohn's disease, celiac disease, systemic lupus erythematosus, ankylosing spondylitis, primary biliary cirrhosis, systemic sclerosis, juvenile arthritis, and Grave's disease. All show an association with at least 1 of these diseases with $P \leq$ $5 \times 10^{-8.27}$ 


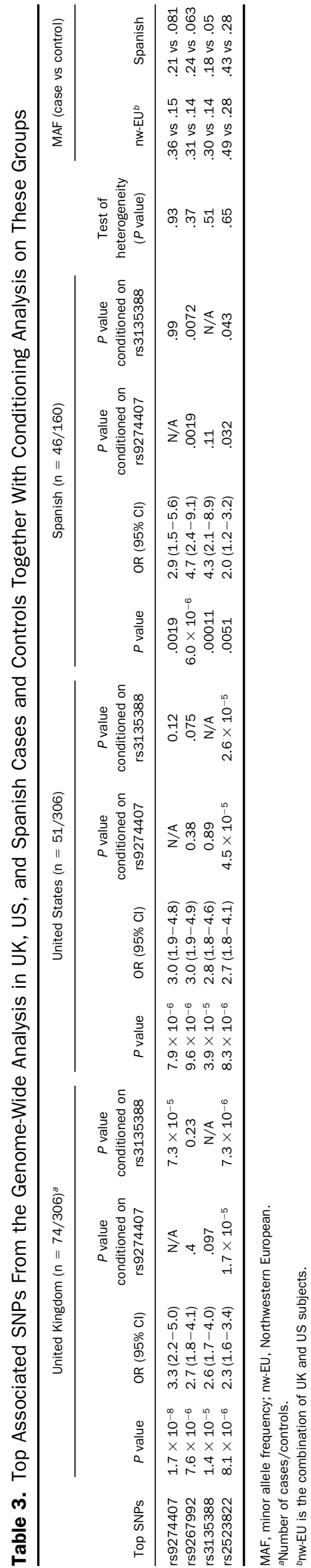

erogeneity on the effect of $A^{*} 0201$ gave a $P$ value of .071 , suggesting a population-dependent effect (Supplementary Figure 4). This inconsistency with the result of rs 2523822 could be explained by the much lower LD between rs2523822 and $A^{*} 0201$ in Spanish cases (Supplementary Table 3). Additionally, $B * 1801$ was associated in Spanish cases with nominal significance independently of $A^{*} 0201$ and $D Q B 1{ }^{*} 0602$ but not in Northwestern Europeans.

The frequencies of the 5-gene HLA haplotypes in cases and controls are shown in Supplementary Table 4. The most significant association was observed for $A^{*} 0201$ $B * 0702-D R B 1 * 1501-D Q B 1 * 0602$ in Northwestern Europeans $(P=.00074$; OR, 13) and Spanish $(P=.013$; OR, 20). However, both $D Q B 1 * 0602$ and $A^{*} 0201$ were significantly associated conditioned on that haplotype. $A * 0201-B * 1801$ was significantly associated $(P=.015$; OR, 6.4) in Spanish only.

\section{Relationship of Genotype With Clinical Features}

We investigated the relationship between the top associated HLA alleles and clinical features of AC-DILI, including age at onset, pattern of liver damage, and disease severity as assessed by magnitude of transaminase or bilirubin elevation (Supplementary Table 5). B*1801 carriage was significantly correlated with peak ALT values in Spanish $(P=.0056$, Figure $3 A)$ but not in Northwestern Europeans $(P=.90)$. We further investigated whether the effect of $B^{*} 1801$ varied in Spanish cases according to pattern of liver damage (Figure $3 B$ ). The estimated effect in hepatocellular cases $(\mathrm{OR}, 8)$ was much larger than cholestatic/mixed cases (OR, 2), but the apparent heterogeneity was not statistically significant $(P=.10)$. No other notable correlations were observed in either Spanish or Northwestern Europeans.

\section{Clinical Predictive Values}

Assuming the prevalence of AC-DILI is $0.014 \%{ }^{2}$ the best positive predictive value is $0.1 \%$ for Northwestern Europeans based on the presence of both $A^{*} 0201$ and $D Q B 1 * 0602$ (frequency of $41 \%$ in cases) and $0.13 \%$ for Spanish based on the presence of both $B^{*} 1801$ and $D Q B 1 * 0602$ (frequency of $8.5 \%$ in cases). In each case, these alleles are present at approximately 10 -fold higher rates than in the population overall. The best negative predictive value (NPV), expressed for clarity as 1-NPV, is $0.006 \%$ for Northwestern Europeans based on the absence of $A^{*} 0201$ (carriage frequency $74 \%$ in cases) and $0.007 \%$ for Spanish based on the absence of rs2523822/C (carriage frequency $74 \%$ in cases), approximately half the assumed rate of AC-DILI in the population overall (Supplementary Table 6).

\section{Discussion}

This study was accomplished through an international cooperation to assemble a considerably larger and more diverse AC-DILI patient collection than previous 
Table 4. Analysis of Combined SNP and HLA Data by Conditioning on $D Q B 1 * 0602$

\begin{tabular}{|c|c|c|c|c|c|c|c|}
\hline \multirow{2}{*}{$\begin{array}{l}\text { Conditioned on } \\
\text { PC1 + PC2 + sex, } \\
\text { and: }\end{array}$} & \multirow[b]{2}{*}{ Allele } & \multicolumn{2}{|c|}{$\begin{array}{c}\text { All } \\
\text { (177 cases, } 219 \text { controls) }\end{array}$} & \multicolumn{2}{|c|}{$\begin{array}{l}\text { Northwestern European } \\
\text { (130 cases, } 107 \text { controls) }\end{array}$} & \multicolumn{2}{|c|}{$\begin{array}{c}\text { Spanish } \\
\text { (47 cases, } 121 \text { controls) }\end{array}$} \\
\hline & & OR $(95 \% \mathrm{Cl})$ & $P$ value & OR $(95 \% \mathrm{Cl})$ & $P$ value & OR $(95 \% \mathrm{Cl})$ & $P$ value \\
\hline & $D Q B 1 * 0602$ & $4.2(2.7-6.6)$ & $4.6 \times 10^{-10}$ & $3.3(2.0-5.7)$ & $1.4 \times 10^{-6}$ & $7.4(3.1-17.8)$ & $7.4 \times 10^{-6}$ \\
\hline$+D Q B 1 * 0602$ & $D R B 1 * 1501$ & $0.8(0.1-5)$ & .72 & $1.4(0.2-10)$ & .77 & $N / A$ & .26 \\
\hline$+D Q B 1 * 0602$ & $D Q A 1 * 0102$ & $2.0(1.2-4.2)$ & .02 & $2.9(1.1-7.2)$ & .02 & $1.8(0.7-5)$ & .37 \\
\hline$+D Q B 1 * 0602$ & $D Q B 1 * 0402$ & $2.3(0.9-6)$ & .1 & $7.5(1.5-38)$ & $4.9 \times 10^{-3}$ & $0.9(0.1-6)$ & .4 \\
\hline$+D Q B 1 * 0602$ & $A * 0201$ & $2.2(1.6-3.2)$ & $1.9 \times 10^{-6}$ & $2.9(1.8-4.4)$ & $7.9 \times 10^{-7}$ & $1.6(0.88-2.8)$ & .13 \\
\hline$+D Q B 1 * 0602$ & $B * 1801$ & $2.0(1.0-3.9)$ & .05 & $0.98(0.36-2.6)$ & .95 & $4.0(1.5-11)$ & .004 \\
\hline$+D Q B 1 * 0602$ and $A * 0201$ & rs2523822/C & $3.9(1.5-11)$ & .01 & $0.86(0.15-4.9)$ & .86 & $7.9(2.1-30)$ & .004 \\
\hline$+D Q B 1 * 0602$ and $A * 0201$ & rs9274407/Minor & $2.4(0.94-5.9)$ & .07 & $9.7(1.9-49)$ & $1 \times 10^{-3}$ & $0.67(0.11-4.1)$ & .65 \\
\hline+ rs9274407/Minor & $D Q B 1 * 0602$ & $1.8(0.68-5.0)$ & .23 & $0.37(0.071-1.9)$ & .2 & $11(1.6-85)$ & .01 \\
\hline
\end{tabular}

PC, principal component.

published studies on the subject. ${ }^{11-13}$ This resulted in the most powerful and comprehensive investigation into genetic risk factors for AC-DILI and the largest GWA for any rare serious adverse event conducted to date. We con-
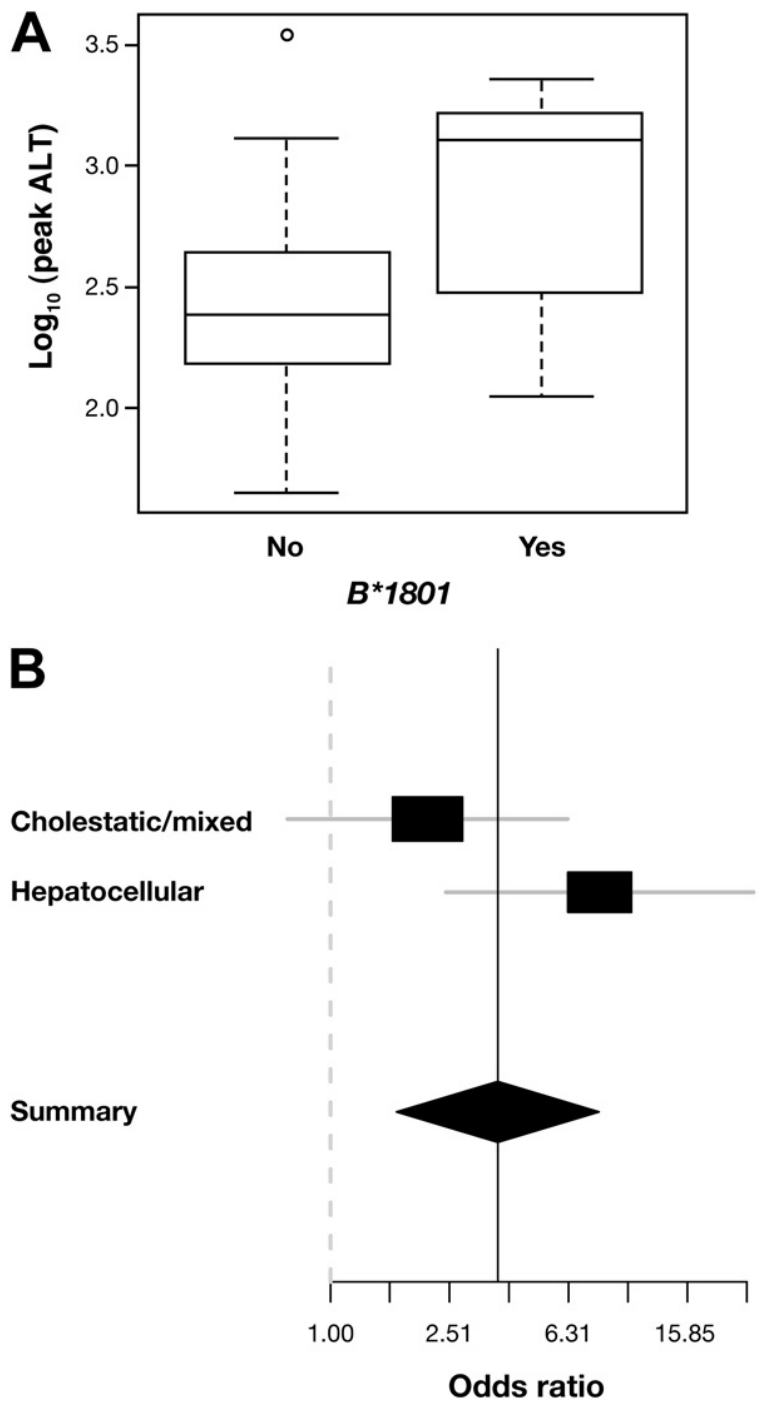

Figure 3. Relationship between HLA genotype and clinical parameters. Panel $A$ shows the relationship between HLA- $B^{\star} 1801$ and peak ALT in Spanish cases and panel $B$ a comparison of odds ratios for different liver damage patterns for HLA-B*1801 in Spanish cases and controls. firmed the previous associations of $H L A-D R B 1 * 1501$ and $D Q B 1 * 0602$ with DILI susceptibility, and our larger sample size and GWA approach allowed the identification of additional HLA risk factors together with an apparent statistical interaction between 2 HLA alleles.

In particular, a novel contribution to disease susceptibility from a common variant ( $\mathrm{rs} 2523822)$ in the region of the HLA-A locus was found. This variant is a tag for the $H L A-A * 0201$ allele in individuals of European ancestry, ${ }^{25}$ and the effect of rs 2523822 and $A^{*} 0201$ were indistinguishable in the Northwestern European subset of this study. When considered as an individual risk factor, the effect of $A^{*} 0201$ was seen only in cases of Northwestern European, and not Spanish, origin. This is in spite of the most strongly associated SNP in this region (rs2523822) showing an effect of similar magnitude in both populations. Nevertheless, the OR for $A^{*} 0201$ in the Spanish subset was also in the same direction as for the other subjects. Comparison of the LD patterns in Northwestern vs Southern European subsets showed that rs2523822 is indeed strongly correlated with $A^{*} 0201$ in Northwestern Europeans $\left(r^{2}=0.96\right)$ but not in Spanish $\left(r^{2}=0.64\right)$. Given this observation, and the high degree of functional variation in the MHC region, one plausible explanation is that $A^{*} 0201$ is not the actual causal variant underlying the association with rs 2523822 but merely happens to be an adequate "tag" for the causal site(s) in Northwestern Europeans but not in the Spanish. Similarly, because the most significant class II association in Northwestern Europeans rs9274407 was still significantly associated conditioned on $H L A-D R B 1 * 1501-D Q B 1 * 0602$ (or its tag SNP rs3135388) but the opposite was not true: there is a possibility that the causal variant(s) may lie in another class II locus. Further investigation of genetic variation in the MHC region and functional assessment of these variants in the context of AC-DILI will be necessary to identify the causal site(s) securely. The differences among populations in terms of risk associated with class I HLA alleles are somewhat similar to the situation with serious skin rash induced by antiepileptic drugs in East Asians where $H L A-B^{*} 1502$ is a risk factor in Han Chinese but not in other groups such as Japanese. ${ }^{29}$ The complexity of the MHC region in terms of the number of variable sites, and 
local differences in $\mathrm{LD}$, make definitive claims of causality particularly difficult.

A relatively rare HLA haplotype positive for $D R B 1 * 1501$ together with $D Q B 1{ }^{*} 0602, A^{*} 0201$, and $B^{*} 0702$ was significantly associated with AC-DILI in both Northwestern Europeans and Spanish. However, conditioning analysis showed that the association was driven by the $A^{*} 0201$ and the $D R B 1 * 1501-D Q B 1 * 0602$ alleles regardless of the cis or trans configuration. We found compelling evidence of a statistical interaction between rs9274407 and rs 2523822 (or $D Q B 1 * 0602$ and $A^{*} 0201$ ). An interaction of this nature appears biologically plausible in view of the complementary roles for the class I and class II HLA gene products in the T-cell response. If validated, this demonstrates the kind of gene-by-gene interaction that has often been speculated on but rarely observed in studies of complex traits in humans. ${ }^{30}$ On the other hand, without knowing the true causal variants, especially for the class I allele, we cannot exclude the possibility that the interaction between classes I and II is due to imperfect tagging of the causal variants by SNPs or classical HLA alleles.

We found evidence for an increased frequency of $D Q B 1 * 0402$ in the Northwestern European cases. This allele is associated with increased susceptibility to the autoimmune liver disease, primary biliary cirrhosis (PBC). ${ }^{31}$ PBC occurs predominantly in females, whereas a majority of the DILI patients positive for $D Q B 1 * 0402$ in this study were males and therefore unlikely to be misdiagnosed PBC cases. Xenobiotic exposure is believed to have a role in triggering $\mathrm{PBC},{ }^{32}$ and it is possible that there are common susceptibilities to PBC and AC-DILI.

From previous reports, it appears that the HLA class II $D R B 1 * 1501-D Q B 1 * 0602$ haplotype is also a risk factor for other forms of DILI. ${ }^{14,33}$ A second HLA class II haplotype, $D R B 1 * 0701-D Q A 1 * 0201$, is a risk factor for hepatotoxicity relating to ximelagatran ${ }^{34}$ and lapatinib. ${ }^{35}$ However, apart from our reported association between flucloxacillin DILI and $B^{*} 5701^{16}$ and an association between $A^{*} 3303$ and ticlopidine DILI in Japanese, ${ }^{36}$ the role of HLA class I genotypes in susceptibility to DILI generally is still poorly understood and merits further investigation. Interestingly, in addition to being possibly associated with DILI in the Spanish, $B^{*} 1801$ was further associated with peak ALT values in these cases, suggesting a possible role for this allele in phenotypic expression. It is worth noting that the only 2 cases of fulminant liver failure in the Spanish cohort were both $B^{*} 1801$ carriers.

Additionally, the possibility that the genetic determinants of DILI risk detected here using common SNP markers may potentially represent contributions from a larger number of rare genetic variants should be considered. ${ }^{37}$ This is particularly relevant to rare adverse events such as DILI, for which rare genetic determinants are in principle one of the most parsimonious explanations. Detection of rare variants will require whole genome sequencing, which is increasingly feasible. ${ }^{38}$

Although our GWA study failed to provide any genome-wide significant evidence of a role for non-HLA genes, including ADME genes, in AC-DILI susceptibility, it remains possible that other genes contribute with smaller effects. In particular, the connection between ACDILI and autoimmune disease is further extended by the association of the PTPN22 SNP rs2476601. This association could also apply to autoimmune-related DILI because of other drugs because the gene product has a general role in regulation of $\mathrm{T}$-cell responses.

This unique study has demonstrated the ability to determine novel genetic risk factors of AC-DILI in diverse populations. We have shown there are improvements in the NPV for the combined HLA risk genotypes compared with our assumed incidence of AC-DILI of $0.014 \%$ with carriers of both class I and II risk alleles showing a nearly 10 -fold increase in risk. A positive predictive value of just $0.1 \%$ means that HLA genotyping will not be an effective means of prospectively identifying those at risk of ACDILI, but our findings have clinical utility in that HLA genotyping may be of value in strengthening AC-DILI diagnoses in view of the high NPVs seen.

\section{Supplementary Material}

Note: To access the supplementary material accompanying this article, visit the online version of Gastroenterology at www.gastrojournal.org, and at doi: 10.1053/j.gastro.2011.04.001.

\section{References}

1. Kaplowitz N. Idiosyncratic drug hepatotoxicity. Nat Rev Drug Discov 2005;4:489-499.

2. Sgro $C$, Clinard F, Ouazir K, et al. Incidence of drug-induced hepatic injuries: a French population-based study. Hepatology 2002;36: 451-455.

3. Ostapowicz G, Fontana RJ, Schiodt FV, et al. Results of a prospective study of acute liver failure at 17 tertiary care centers in the United States. Ann Intern Med 2002;137:947-954.

4. Stricker BH, Van den Broek JW, Keuning J, et al. Cholestatic hepatitis due to antibacterial combination of amoxicillin and clavulanic acid (augmentin). Dig Dis Sci 1989;34:1576-1580.

5. de Abajo FJ, Montero D, Madurga M, et al. Acute and clinically relevant drug-induced liver injury: a population based case-control study. Br J Clin Pharmacol 2004;58:71-80.

6. Andrade RJ, Lucena MI, Fernandez MC, et al. Drug-induced liver injury: an analysis of 461 incidences submitted to the Spanish registry over a 10-year period. Gastroenterology 2005;129:512521.

7. Chalasani N, Fontana RJ, Bonkovsky HL, et al. Causes, clinical features, and outcomes from a prospective study of drug-induced liver injury in the United States. Gastroenterology 2008;135: 1924-1934,e1-e4.

8. Andrade RJ, Lucena MI, Kaplowitz N, et al. Outcome of acute idiosyncratic drug-induced liver injury: long-term follow-up in a hepatotoxicity registry. Hepatology 2006;44:1581-1588.

9. Garcia Rodriguez LA, Stricker BH, Zimmerman HJ. Risk of acute liver injury associated with the combination of amoxicillin and clavulanic acid. Arch Intern Med 1996;156:1327-1332.

10. Fontana RJ, Shakil AO, Greenson JK, et al. Acute liver failure due to amoxicillin and amoxicillin/clavulanate. Dig Dis Sci 2005;50: 1785-1790.

11. Hautekeete ML, Horsmans $Y$, van Waeyenberge $C$, et al. HLA association of amoxicillin-clavulanate-induced hepatitis. Gastroenterology 1999;117:1181-1186. 
12. O'Donohue J, Oien KA, Donaldson P, et al. Co-amoxiclav jaundice: clinical and histological features and HLA class II association. Gut 2000;47:717-720.

13. Donaldson PT, Daly AK, Henderson J, et al. Human leucocyte antigen class II genotype in susceptibility and resistance to coamoxiclav-induced liver injury. J Hepatol 2010;53:1049-1053.

14. Andrade RJ, Lucena MI, Alonso A, et al. HLA class II genotype influences the type of liver injury in drug-induced idiosyncratic liver disease. Hepatology 2004;39:1603-1612.

15. Lucena MI, Andrade RJ, Fernandez MC, et al. Determinants of the clinical expression of amoxicillin-clavulanate hepatotoxicity: a prospective series from Spain. Hepatology 2006;44:850-856.

16. Daly AK, Donaldson PT, Bhatnagar P, et al. HLA-B*5701 genotype is a major determinant of drug-induced liver injury due to flucloxacillin. Nat Genet 2009;41:816-819.

17. Molokhia M, McKeigue P. EUDRAGENE: European collaboration to establish a case-control DNA collection for studying the genetic basis of adverse drug reactions. Pharmacogenomics 2006;7: 633-638.

18. Fontana RJ, Watkins PB, Bonkovsky HL, et al. Drug-Induced Liver Injury Network (DILIN) prospective study: rationale, design and conduct. Drug Saf 2009;32:55-68.

19. Danan G, Benichou C. Causality assessment of adverse reactions to drugs. I. A novel method based on the conclusions of international consensus meetings: application to drug-induced liver injuries. J Clin Epidemiol 1993;46:1323-1330.

20. Nelson MR, Bryc K, King KS, et al. The Population Reference Sample, POPRES: a resource for population, disease, and pharmacological genetics research. Am J Hum Genet 2008;83:347358.

21. Purcell S, Neale B, Todd-Brown K, et al. PLINK: a tool set for whole-genome association and population-based linkage analyses. Am J Hum Genet 2007;81:559-575.

22. Browning SR, Browning BL. Rapid and accurate haplotype phasing and missing-data inference for whole-genome association studies by use of localized haplotype clustering. Am J Hum Genet 2007; 81:1084-1097.

23. The R Project for Statistical Computing. Available at: http://www. r-project.org. Accessed March 9, 2011.

24. Novembre J, Johnson T, Bryc K, et al. Genes mirror geography within Europe. Nature 2008;456:98-101.

25. de Bakker PI, McVean G, Sabeti PC, et al. A high-resolution HLA and SNP haplotype map for disease association studies in the extended human MHC. Nat Genet 2006;38:1166-1172.

26. Ahmadi KR, Weale ME, Xue ZY, et al. A single-nucleotide polymorphism tagging set for human drug metabolism and transport. Nat Genet 2005;37:84-89.

27. Hindorff LA, Junkins HA, Mehta JP, et al. A catalog of published genome-wide association studies. Available at: www.genome.gov/ gwastudies/. Accessed March 9, 2011.

28. Chung SA, Criswell LA. PTPN22: its role in SLE and autoimmunity. Autoimmunity 2007;40:582-590.

29. Kaniwa N, Saito Y, Aihara M, et al. HLA-B locus in Japanese patients with anti-epileptics and allopurinol-related Stevens-Johnson syndrome and toxic epidermal necrolysis. Pharmacogenomics 2008;9:1617-1622.

30. Moore JH, Williams SM. Epistasis and its implications for personal genetics. Am J Hum Genet 2009;85:309-320.

31. Hirschfield GM, Liu X, Xu C, et al. Primary biliary cirrhosis associated with HLA, IL12A, and IL12RB2 variants. N Engl J Med 2009; 360:2544-2555.

32. Selmi C, Gershwin ME. The role of environmental factors in primary biliary cirrhosis. Trends Immunol 2009;30:415-420.
33. Singer JB, Lewitzky S, Leroy E, et al. A genome-wide study identifies HLA alleles associated with lumiracoxib-related liver injury. Nat Genet 2010;42:711-714.

34. Kindmark A, Jawaid A, Harbron CG, et al. Genome-wide pharmacogenetic investigation of a hepatic adverse event without clinical signs of immunopathology suggests an underlying immune pathogenesis. Pharmacogenomics J 2008;8:186-195.

35. Spraggs CF, Budde LR, Briley LP, et al. HLA-DQA1*02:01 is a major risk factor for lapatinib-induced hepatotoxicity in women with advanced breast cancer. J Clin Oncol 2011;29:667-673.

36. Hirata K, Takagi H, Yamamoto M, et al. Ticlopidine-induced hepatotoxicity is associated with specific human leukocyte antigen genomic subtypes in Japanese patients: a preliminary case-control study. Pharmacogenomics J 2008;8:29-33.

37. Dickson SP, Wang K, Krantz I, et al. Rare variants create synthetic genome-wide associations. PLoS Biol 2010;8:e1000294.

38. Ashley EA, Butte AJ, Wheeler MT, et al. Clinical assessment incorporating a personal genome. Lancet 2010;375:1525-1535.

Received January 14, 2011. Accepted April 1, 2011.

\section{Reprint requests}

Address requests for reprints to: Ann K. Daly, PhD, Institute of Cellular Medicine, Newcastle University Medical School, Framlington Place, Newcastle upon Tyne NE2 4HH, United Kingdom. e-mail: a.k.daly@ncl.ac.uk; fax: (44) 1912220723.

\section{Acknowledgments}

Contributors to sample collection via Spanish DILI network, EUDRAGENE, DILIN, and DILIGEN are listed in the Appendix.

Drs Lucena, Molokhia, Shen, and Daly contributed equally to this manuscript.

\section{Conflicts of interest}

The authors disclose the following: Dr Fontana acted as a consultant to GlaxoSmithKline. Dr Nelson is an employee of GlaxoSmith Kline. The remaining authors disclose no conflicts.

\section{Funding}

The genome-wide association study and HLA genotyping was funded by the International Serious Adverse Events Consortium with support from Abbott, Daiichi-Sankyo, GlaxoSmithKline, Johnson \& Johnson, Novartis, Pfizer, Roche, Sanofi-Aventis, Takeda, Wellcome Trust, and Wyeth. The DILIGEN sample collection was partly funded by the UK Department of Health (ref. PHGX10A) and by UK NIHR funding to the Nottingham Digestive Diseases Centre. The DILIN network is structured as a U01 cooperative agreement with funds provided by the National Institute of Diabetes and Digestive and Kidney Diseases (NIDDK) under grants 2U01-DK065176-06 (Duke), 2U01-DK065201-06 (UNC), 2U01-DK065184-06 (Michigan), 2U01-DK065211-06 (Indiana), 5U01DK065193-04 (UConn), 5U01-DK065238-08 (UCSF/CPMC). Additional funding is provided by CTSA grants UL1 RR025761 (Indiana), UL1 RR025747 (UNC), and UL1 UL1 RR024986 (UMich). The EUDRAGENE collaboration has received support from the EC 5th Framework program (QLRI-CT-200202757) and the SAEC. The UK NIHR postdoctoral award funded Mariam Molokhia. The Spanish DILI Registry is partly funded by the Spanish Medicine Agency and has received support from SAEC and Boehringer-Ingelheim, Barcelona, Spain. CIBERehd and RETIC are funded by Instituto de Salud Carlos III. The funders had no role in study design, data collection and analysis, decision to publish, or preparation of the manuscript. 